\title{
Los factores socioculturales, teleformación y Tecnologia de la Información y Comunicación
}

\author{
Sociocultural factors, Network communication and Information Technology and \\ Communication
}

\section{Cornelio Comet Weiler ${ }^{1}$ y Luis Ortiz Jiménez ${ }^{2}$}

Resumen: Paraguay es un pais que tiene una cultura homogeneizada por su gran ascendencia hispano-guaraní y el fuerte predominio de su lengua guaraní; esta diversidad personal y cultural, contribuye a enriquecer la convivencia. Un punto crucial y preocupante son las desigualdades existentes en la sociedad paraguaya, constituyéndose en una de las principales causales de los problemas tanto en el ámbito social como educativo y esto se enmarca en la gran brecha para acceder a los avances tecnológicos que impone la globalización a nivel mundial, que además de los requerimientos de programas informáticos y/o técnicos, implica también todo un cambio de cultura. El acceso a programas informáticos ya constituye todo un cambio de cultura, el cual se está imponiendo. Para ello, es importante adentrarnos a la Tecnología de la Información y Comunicación (TIC) y la teleformación en nuestro contexto paraguayo que conlleva la realidad de la coyuntura sociocultural referido sobre todo a creencias y realidades.

Palabras clave: Teleformación, brecha, sociedad, cultura, TIC.

Abstract: Paraguay is a country that has a homogenized culture for its great Hispanic-Guarani influence and the strong predominance of the Guarani language; this personal and cultural diversity enriches coexistence. A crucial and worrying point about the inequalities existing in Paraguayan society, becoming one of the main causes of the problems in the social sphere as well as in education and this is part of the large gap for access to the technological advances that globalization imposes worldwide that, in addition to the requirements of computer and/or technical programs, also involves a whole culture change. Access to software programs it is already a complete change of culture, which is being imposed. To reach this, it is important to delve the Information and communications technology (ICT) and the network communication to our Paraguayan context which involves socio-cultural reality of the situation primarily concerned with beliefs and realities.

Keywords: Network communication, gap, society, culture, ICT.

${ }^{1}$ Doctorando en Ciencias de la Educación por la Universidad de Almería, España. Catedrático en Métodos de la Investigación de la Universidad Autónoma de Asunción, Paraguay. E-mail: cometweiler@gmail.com 2 Doctor en Ciencias de la Educación, por la Universidad de Granada. Profesor de tiempo completo en el Departamento de Educación de la Universidad de Almeria. España. E-mail: lortizj@ual.es

Recibido: 16/03/2015; Aceptado: 11/06/2015. http://dx.doi.org/10.18004/riics.2015.julio.86-101

Rev. Int. Investig. Cienc. Soc.

Vol. $11 \mathrm{n}^{\circ} 1$, julio 2015. pág. 86-101.

ISSN (Impresa) 2225-5117. ISSN (En Línea) 2226-4000. 


\section{INTRODUCCIÓN}

El tema del desarrollo de la tecnología en los últimos tiempos, se evidencia a nivel mundial y este avance se ha insertado, no sólo como un estatus o indicador de desarrollo, sino como una necesidad. La inserción de la tecnología, y de una manera especial la Tecnología de Información y Comunicación (TIC), no tuvo la misma magnitud para todos los países, pues diferentes aspectos han influido para ello: la cultura, el desarrollo económico y social, la política de desarrollo del país, la apertura y el grado de democratización de los estados, la relación economía y costo de la infraestructura para implementar una tecnología avanzada, el mismo costo de las TIC, entre otros. Esto por mencionar solo algunos elementos que han tenido gran influencia, sobre todo en los países en vías de desarrollo y subdesarrollados.

Si bien Paraguay cuenta con distintos tipos de tecnologías para la conexión a internet, el costo es aún alto. Existe una diferencia notoria con el resto de los países de la región del Mercosur, pues el avance de las TIC es inferior a ellos. En una entrevista realizada por un periódico local por el periodista Oscar Bogado (Diario última Hora) al Secretario General de la Cámara Paraguaya de Internet (CAPADI), Rubén González, en el año 2012, en referencia a los costos y servicios de Internet en la Región, mencionaba: “[...]. En comparación con otros países el Paraguay actualmente paga unos 20,23\$ por 1 Mbps, Argentina 15,59\$ y Brasil $17,89 \&[\ldots]$ ]. Hay una variación, no sólo entre los países entre sí, sino también dentro de cada país. Para el 2014, acorde a la firma Akamai Technologies, Paraguay sigue teniendo un crecimiento lento en cuanto a los servicios de internet encontrándose por debajo del promedio mundial, sin embargo entre el año 2012 y el 2013, aumenta del 14\% hasta llegar a 1,5 Mbps" (ABC color, nacionales, 20/02/2014). En enero del 2015, Iriarte Denis mencionaba, que en el último lugar en cuanto a ranking en Sudamérica, se encontraba Paraguay con 2,61 Mbps (Periódico económico digital 5 días).

Lo mencionado, es importante ya que el desarrollo de la tecnología está identificada con el progreso y las posibilidades de lograr calidad de vida, sobre todo cuando está al servicio de la población y que estas puedan tener accesibilidad a ella, pero debemos reconocer que existe una gran brecha digital, realidad que llevará tiempo acortarse entre los países desarrollados y los menos desarrollados, donde están incluidas las tecnologías de redes, telecomunicaciones e informática (teléfono, televisión, radio, Internet, computadoras, etc.), que de manera directa o indirecta, influyen en nuestras actividades socioeconómicas, educativas y culturales (Martínez Serrano y Serrano Santoyo, 2007). 
Es realmente asombrosa la evolución de la tecnología, que insertada dentro de los procesos y sistemas de la educación en cualquiera de los niveles exige y exigirá una formación continua de quienes estén involucrados en su aplicación, como bien lo manifiestan Navales, Omaña y Perazzo (2000), haciendo referencia a que la universidad debiera formar estos recursos humanos con calidad no solamente en un ámbito de conocimiento, sino que van más allá dándole también el condimento en el afán de no ser simples importadores y consumidores de información y tecnologias.

Maldonado López (2007, p. 261), es bien explicita al enfocar su análisis en el desarrollo de las tecnologías de la comunicación al indicar a las TIC como "principales factores externos de cambio en las universidades, en particular sobre el impacto de las tecnologías de la información y las comunicaciones en los cuatro ámbitos principales de la actividad universitaria: docencia, investigación, gestión y presencia en el entorno social".

Dentro de esta línea mencionada, la educación a distancia, posibilitada por este avance de las TIC, ha revolucionado algunas facetas de la educación formal y a la no formal, abriendo las puertas a innumerables posibilidades de acceso al conocimiento, teniendo en cuenta que, como en cualquier proceso educativo, coexisten las ventajas y desventajas.

La teleformación, en especificidad la educación a distancia, es un vocabulario "quizá hasta novedoso" aún en el Paraguay y no muy bien comprendido en cuanto a su magnitud en la educación y desarrollo del país. Traigo a colación, para respaldar la importancia de esta temática, dos ideas de lo expuesto en la declaración mundial sobre la educación Superior en el siglo XXI:

[...La educación superior debe hacer frente a la vez a los retos que suponen las nuevas oportunidades que abren las tecnologías, que mejoran la manera de producir, organizar, difundir y controlar el saber y de acceder al mismo. Deberá garantizarse un acceso equitativo a estas tecnologías en todos los niveles de los sistemas de enseñanza (UNESCO, 1998, p.30).

Este texto, ya en 1998, reafirma la importancia de una tecnología accesible para la expansión de la educación, específicamente la superior, pues la brecha de oportunidades de formación y acceso a la tecnología entre los países subdesarrollados y en vías de desarrollo respecto a los países industrializados es abismal. Al respecto, Urkola Carrera (2006, p. 40) recalca que una tecnología "no sólo tiene implicaciones sociales, sino que también es producto de unas 
determinadas condiciones sociales y económicas de una época y un lugar, es decir, que es producto de una construcción social".

Es por ello que para hacer posible que la Tecnología sea un instrumento apto para enfrentar algunos de los desafios que las sociedades tienen por delante, es preciso que haya varias decisiones politicas-sociales que deben ser tomadas con una mirada estratégica, como ser la perspectiva de innovación, fortalecimiento de la capacidad científica y tecnológica, vinculación de actores, una educación que rompa el analfabetismo tecnológico, achicamiento de la brecha digital en el país, entre otros.

A raíz de esta evolución tecnológica, donde el manejo electrónico de la información es la clave, se originó una era en la que el conocimiento es el recurso y punto clave más importante para el desarrollo de una sociedad.

La teleformación, está aún "en pañales” en el país, quizás las primeras experiencias en esta área en educación superior no hayan sido de las más auspiciosas, ya que algunas estrategias utilizadas como plataforma de lanzamiento incidieron en la calidad y en la "confianza hacia ella" del potencial alumnado y de la sociedad misma hacia este estilo: la calidad de los formatos escritos que fungieron de guías en un primer momento; la precariedad y el alto costo de Internet; plataformas de Intranet (utilizada inicialmente) no muy amigables y manejadas precariamente por algunos docentes y alumnos; la dificultad de acortar distancias entre universidad y alumnado; docentes con una formación deficitaria en esta línea; las mismas universidades que no dieron el apoyo y voto de confianza desde el inicio para que esta modalidad tome vuelo produciéndose a raíz de esto deserciones en el profesorado y alumnado y, en casos, alumnos que quedaron a media carrera por que las universidades no encontraron respuestas y soluciones adecuadas a las situaciones adversas que se fueron presentando y en algunos casos, se les han cerrado las puertas o dándoseles soluciones a medias.

Para que este estilo de formación llegue a implementarse eficazmente en el país, es importante determinar y tener en cuenta algunos elementos que inciden en este paulatino proceso y que permiten una visión de la realidad a modo de conocer el cómo encararlo y a qué atenerse, a fin de que los resultados sean los esperados. En este sentido GATE, en 1998, afirmaba que:

Las universidades llevan tiempo siendo conscientes de que las enseñanzas que tradicionalmente vienen impartiendo no se corresponden con las necesidades de toda la sociedad y que el colectivo objeto de su atención no debe reducirse a los jóvenes estudiantes procedentes del bachillerato, sino que también debe 
incluir adultos y profesionales de las empresas. Además, no parece posible dotar a las personas de capacidades estratégicas exclusivamente en el periodo que dura su formación en el sistema educativo tradicional. La sociedad actual demanda una educación y formación mucho más flexible y abierta a lo largo de toda la vida profesional "Lifelong Learning" (p. 38).

La educación universitaria en Paraguay ha tomado impulso a partir del cambio de rumbo del país, pasando del modelo dictatorial a la democrática acaecido en 1989; más este cambio fue más en cantidad que en calidad. Ante esto hubo constantes y duras críticas de algunos sectores sobre la necesidad de una reforma de la educación superior en sí y de su estructura, premisa que duró largos años y desencadenó un debate cuyo fruto fue la recientemente Ley de Educación Superior N4995/2013 sancionado por el poder Legislativo en el año 2013. La transformación, a partir de esto, de nuestro sistema educativo superior, incidirá no sólo en la mejora de la misma, sino en esta sociedad en constante evolución.

Dugarte de Villegas y Guanipa (2009) refieren el fuerte efecto de los cambios en el ámbito educativo superior, ya que las universidades, como los institutos superiores son responsables de la formación profesional de jóvenes y adultos que deberán insertarse y responder a los desafios de una sociedad cada vez más exigente, empapada y sedienta a la vez del conocimiento, de la información y enfocada a buscar y mejorar la calidad de vida.

\section{El impacto de las TIC y la brecha digital en el Paraguay}

Marqués Graells (2000), menciona que la sociedad en que estamos insertos, evoluciona acompañada de los avances científicos, en un escenario globalizado y apoyado por potentes y versátiles TIC y “...sus efectos se manifiestan de manera muy especial en las actividades laborales y en el mundo educativo...”. En estos términos, Martínez Sánchez (2001) menciona que:

[...Junto con esta posibilidad, las nuevas tecnologías tienen otra faceta, quizás la socialmente más conocida; su utilización como un instrumento de trabajo, como una herramienta que puede estar presente en la práctica totalidad del quehacer humano, facilitando el mismo y potenciándolo, tanto por lo que supone al requerir un menor esfuerzo físico, como por el desarrollo de trabajo que permite. Los dos aspectos son razones más que suficientes para su inclusión como función diferenciada...] (p. 204). 
Tal como se expresa en estos párrafos, a nivel internacional se observa una evolución sorprendente de la enseñanza virtual, quizá como fruto de una madurez en la búsqueda de un contexto en donde la educación cobre un nuevo y atrayente protagonismo y es por ello que la teleformación en la modalidad e-learning, ostente hoy día como una estrategia de educación y formación flexible, aceptado y que se adapta a los distintos ámbitos que la sociedad plantea, como ser en la educación, salud, empresas, en el trabajo, en las gestión estatal y privada, cultura y en otros ámbitos con todos sus niveles y modalidades.

Por otra parte, Peterssen Nodarse (2001) menciona algunos escollos o barreras, los cuales debieran de superarse para que sea puesta en marcha una enseñanza virtual a través de la Internet. Estas son brechas, aplicables al Paraguay y que obstaculizan cualquier proceso educativo donde se busque que la tecnología de la comunicación y la información sean una herramienta estratégica de mejoramiento y sobre todo en nuestro país donde el desarrollo de la tecnología nos ubica por debajo de cualquier otro país en vía de desarrollo en América Latina. Estas realidades de las que habla Peterssen, se presentan a continuación:

- Los bajísimos niveles de penetración de la informática en la enseñanza primaria y secundaria.

- Los elevados niveles de analfabetismo informático existente en Latinoamérica.

- El alto coste de conexión para los servidores

- La actitud "lejana e indiferente" de los centros de enseñanza y sus protagonistas pedagógicos respecto a la enseñanza virtual a través de internet.

- La ausencia de políticas efectivas que conlleven a la implementación de soluciones informáticas centralizadas en la mayoría de los ministerios de educación y cultura de los países de la región.

A estas barreras podemos mencionar las indicadas por Goñi (2005), las cuales también van muy unidas a las mencionadas anteriormente, estas se dan entre los desajustes producidos por la renovación tecnológica, donde se pueden identificar los siguientes:

- Brecha social: provocada por la falta de formación durante la juventud o por una escasa capacidad económica. 
- Brecha accesibilidad: originada por una menor capacidad sensorial o cognitiva.

- Brecha tecnológica: desactualización producida por la falta de interés por lo novedoso.

- Punto de bifurcación tecnológica: edad en la que se considera innecesario recurrir a nuevas tecnologías.

En la declaración de Asunción de la XXI Cumbre Iberoamericana del 29 octubre 2011 (Rojas, 2013, p. 5-6), se plantearon propuestas y acciones en busca de reducir esa gran diferencia en lo concerniente al acceso a la tecnología:

- Reducir la brecha digital y promover el acceso a capacidades en el uso universal de las TICs, con miras a la consolidación de una sociedad del conocimiento.

- Reducir asimismo la brecha tecnológica mediante el intercambio de conocimientos científicos, técnicos y tecnológicos, así como de experiencias, metodologías y técnicas en tales ámbitos, en el espacio iberoamericano, atendiendo la normativa aplicable.

- Auspiciar el desarrollo del uso de las TICs, de acuerdo con las condiciones y posibilidades de cada país, en todos los niveles del sector público para contribuir a la transparencia, mejora y eficiencia de la gestión, generar mayor participación y competitividad, facilitar el acceso de la ciudadanía a la información, potenciar la calidad de los servicios públicos y promover la demanda de servicios en línea por parte de la ciudadanía.

- Robustecer las redes regionales de gobierno electrónico y la cooperación en este ámbito, atendiendo a la importancia de las TICs como sustento de las etapas de las políticas públicas.

\section{Las TIC en la educación superior del Paraguay}

El uso del computador en el aula actualmente es muy distinto de lo que fue hace 10 o 15 años atrás, que ya no se limita solo a las aulas presenciales ya que las aulas virtuales, aunque pálidamente aún, van ganado espacios dentro del contexto educativo universitario paraguayo. Hoy en día, los estudiantes necesitan desarrollar destrezas para la utilización adecuada de los recursos informáticos y que le puedan ser útiles, es importante ser competentes en el uso de recursos de las TIC. 
La educación a nivel superior tiene muchos recursos para poner en práctica los objetivos a los cuales está enfocado, cual es introducirse en la vida social, laboral y productiva de la sociedad, dándoles ese impulso vital y de calidad, introduciéndose e introduciéndolos en los nuevos parámetros tecnológicos aplicados a la educación. Como menciona Cueva Carrión et al. (2009), esto se enmarca dentro de la necesidad de la educación superior en avanzar cualitativa y cuantitativamente, aspecto que no puede considerarse como una moda o esnobismo.

En manifestaciones de Cernuzzi, Vargas y González (2008), afirman que:

En los últimos años nuestro país ha asistido a un crecimiento sostenido de la difusión de las tecnologías de telefonía móvil y de Internet. En este contexto, todas las universidades tienen acceso a Internet, donde varias de ellas utilizan herramientas digitales para facilitar la interacción entre profesores y estudiantes (Moodle, Claroline, etc.) e inclusive algunas ofrecen cursos de educación a distancia, aunque no existen estudios acerca de la calidad de estas ofertas. Sin embargo, en general en la práctica docente, y por ende en el proceso de enseñanza-aprendizaje, es todavía bastante reducida la utilización de las TIC. Esto limita la posibilidad de fomentar una mayor cultura tecnológica por parte de las nuevas generaciones (p. 24-25).

A partir de estas reflexiones sobre la teleformación, podemos afirmar que la implementación de esta modalidad dentro del sistema educativo superior, la cual se afianza a nivel mundial, se está tornando una necesidad dentro de la realidad universitaria paraguaya, aunque la brecha digital sea una barrera que llevará mucho tiempo superarla.

\section{Sociedad y Cultura. Aspectos incidentes en la penetración digital de la teleformación}

La cultura según Hughes (2007) se refiere a “aquellos comportamientos aprendidos donde se caracteriza la forma de vida total de los miembros dentro de una sociedad determinada" (p. 151). Por otro lado Harris (1990), al definir sociedad hace referencia a una población con una forma de vida organizada.

Es en este escenario donde social y culturalmente hace su inserción la tecnología digital $\mathrm{y}$, en diferentes escenarios, favoreció nuevas formas de organización económica y relaciones socioculturales, traspasando fronteras y desplegando espacios para la conformación de la sociedad de la información y del 
conocimiento. Y en referencia al proceso de penetración, la Organización de las Naciones Unidas, en un material referente a las Tecnologías de la información y las comunicaciones para un desarrollo social y económico incluyente, menciona que la "tasa de adopción de las TIC en las sociedades, durante los últimos dos decenios ha superado la de cualquier otra tecnología anterior. Por consiguiente, también son susceptibles de cambios rápidos las oportunidades y los desafios que suponen las TIC" (ONU, 2014, p. 6).

Por su parte Marqués Graells (2000), propone algunas peculiaridades de la sociedad actual en el siguiente estracto "características de la sociedad actual", las cuales las traigo a colación por "dibujar" al perfil de nuestro mundo actual y globalizado y que está muy relacionado a la temática aquí desarrollada.

Características de la sociedad actual: Aspecto Socioculturales (Marqués Graells (2000. p. 3).

- Continuos avances científicos: Incesantes descubrimientos y nuevos desarrollo científicos: nuevas tecnologías para la información y la comunicación (telefonía, informática, etc.), ingeniería genética, nanotecnología, nuevos materiales...

- Redes de distribución de información de ámbito mundial: Las redes de distribución de información permiten ofrecer en cualquier lugar en el que haya un terminal (ordenador, teléfono móvil, televisor...) múltiples servicios relacionados con la información. No obstante, lo que para algunos países son grandes y veloces "autopistas de la información", para otros apenas son simples caminos de tierra y barro. La sociedad se basa en amplias redes de comunicación y en la capacidad de los individuos para actualizar su conocimiento en un mundo que cambia vertiginosamente.

- Omnipresencia de los medios de comunicación de masas e Internet: Con los "mass media " (prensa, radio, televisión...) e Internet las noticias de, información, formación y ocio llegan cada vez a más personas. La información se mueve casi con absoluta libertad por todas partes; lo que ocurre en un punto del planeta puede verse inmediatamente en todos los televisores del mundo (hay canales de TV -como la poderosa cadena americana CNN <http://www.cnn.com/>, que emiten noticias durante todo el día). Esto supone una verdadera explosión cultural que hace más asequible el conocimiento a los ciudadanos, pero a la vez, crece el agobio por el exceso de información y la sensación de manipulación ideológica por los grupos de poder que a través de los "mass media" configuran la opinión pública y afianzan determinados valores. 
- Nuevos patrones para las relaciones sociales: Las omnipresentes TIC imponen nuevos patrones sobre la gestión de las relaciones sociales: nuevas formas de comunicación interpersonal, nuevos entretenimientos...

- Mayor información del estado sobre los ciudadanos: No solamente son los ciudadanos los que pueden acceder a un mayor volumen de información a través de Internet; el estado aumenta la información de que dispone sobre los ciudadanos (cámaras de vídeo en las calles, centralización de datos en Hacienda...), y está en condiciones de ejercer un mayor control sobre ellos.

- Integración cultural: Tendencia hacia un "pensamiento único" (sobre todo en temas científicos y económicos) debida en gran parte a la labor informativa de los medios de comunicación social (especialmente la televisión), la movilidad de las personas por todos los países del mundo y la unificación de las pautas de actuación que exige la globalización económica. Se va reforzando la sensación de pertenecer a una comunidad mundial, aunque los países más poderosos van imponiendo su cultura (idioma, instrumentos y procesos tecnológicos...) amenazando la identidad cultural de muchos pueblos.

- Aceptación del "imperativo tecnológico": Según el "imperativo tecnológico", la fabricación y utilización de herramientas es el factor determinante del progreso de la humanidad. Por ello se aceptan los nuevos instrumentos como modernos e inevitables, renunciando muchas veces a conducir el sentido de los cambios y del progreso. Como afirma Joana $\mathrm{M}^{\mathrm{a}}$ Sancho "las tecnologias artefactuales, simbólicas y organizativas transforman de manera insospechada no solo el mundo que nos rodea, sino nuestra propia percepción del mismo y nuestra capacidad para controlarlo". "Hay que tener ordenador y saber inglés"

- Formación de megaciudades: La población se agrupa en grandes aglomeraciones urbanas (megaciudades) donde muchas veces son necesarios desplazamientos importantes para ir a los lugares de trabajo y de ocio.

- Baja natalidad (en los paises desarrollados): En los países desarrollados hay una notable baja de natalidad (Unión Europea), en tanto que en algunos países en desarrollo (China) se toman medidas para contener una tasa de crecimiento excesivo. Mientras otros países siguen sin tomar ninguna medida y con unas tasas de natalidad que desbordan sus posibilidades (México, norte de África).

- Nuevos modelos de agrupación familiar: Aumenta el porcentaje de personas que viven solas (solteras, divorciadas, viudas...) y aparecen nuevos modelos de agrupación familiar (monoparental, homosexual...). El patriarcado entre en 
crisis. El alto índice de separaciones y divorcios genera los consiguientes problemas para los hijos.

- Mayor presencia de la mujer en el mundo laboral: Mayor incorporación de la mujer al trabajo, dedicando menos tiempo a la familia. Las posibilidades de actuación social de la mujer se van igualando a las del hombre en la mayoría de los países, aunque en otros (como los países musulmanes) este proceso se ve fuertemente dificultado por sus tradiciones religiosas. En muchos casos la mujer sufre el desgaste de una doble jornada laboral: en el trabajo y como ama de casa.

- Necesidad de "saber aprender" y de una formación permanente: Ante la imposibilidad de adquirir el enorme y creciente volumen de conocimientos disponible, las personas debemos saber buscar autónomamente la información adecuada en cada caso (aprender a aprender). Las nuevas generaciones se van encontrando con muchos conocimientos nuevos respecto a los que presidieron la vida de la generación anterior. Las personas necesitan una formación continua a lo largo de toda la vida para poder adaptarse a los continuos cambios que se producen en nuestra sociedad. No se trata de simples "reciclajes", sino de completos procesos de reeducación necesarios para afrontar las nuevas demandas laborales y sociales.

- Relativismo ideológico: Tendencia a un relativismo ideológico (valores, pautas de actuación...) que proporciona una mayor libertad a las personas para construir su propia personalidad, aunque encuentran a faltar referentes estables sobre los que construir su vida. También emergen nuevos valores que muchas veces se oponen abiertamente a los valores tradicionales, creando desconcierto en una gran parte de la población. Se da gran importancia al momento presente y a la inmediatez (éxito fácil...), al "tener" sobre el "ser"....

- Disminución de la religiosidad: Fuerte disminución del sentimiento religioso y del poder de las iglesias tradicionales, aunque proliferan las sectas pseudoreligiosas. Se refuerzan los planteamientos integristas musulmanes.

- Grandes avances en medicina: Grandes avances en la prevención y terapia sanitaria, consecuencia de los desarrollos científicos en ingeniería genética, nanotecnología, láser... Aumento progresivo de la esperanza de vida.

A partir de este panorama que desarrolla Marqués, podemos inferir que las TIC es fruto del desarrollo científico e influyen a su vez en su evolución, 
contribuyendo al desarrollo socioeconómico y sociocultural modificando el sistema de valores vigente.

La penetración de las TIC en el ámbito social y educativo no ha desacelerado al correr de los tiempos, al contrario esta se hace más vertiginosa y con nuevos ingredientes tecnológicos. Al respecto, Sáez Vacas (1997) señala que existen diversas circunstancias que dificultan su más amplia difusión entre todas las actividades y capas sociales como: problemáticas técnicas; falta de formación; problemas de seguridad; barreras económicas; barreras culturales y sociales. Cabe recalcar que estas circunstancias que impiden la difusión rápida y de calidad de las TIC, mencionada por Sáez en 1997, hoy a 20 años, en nuestro medio observamos que sigue siendo actual.

Robles (2003) mencionando a Wild (1999) explicita que hay un consenso claro de que la cultura tiene que tener una influencia definitiva y bien fuerte en el diseño y el uso de la información, en los sistemas de comunicación y de aprendizaje, así como en su manejo. Es por ello que se ha de tener en cuenta frente a la teleformación aspectos como la predisposición, o las actitudes, la disposición, o la misma frustración de los usuarios, ya que los mismos inciden en su utilización.

Sobre este punto Martin (2012, pp.15-16) indica que la formación en las aplicaciones de las TIC y el cambio cultural son factores decisivos en la integración de las TIC en la educación y puede constituirse clave del proceso para su adopción como herramientta útil y necesaria.

El avance tecnológico y sobre todo lo concerniente a la Información y Comunicación, trajo consigo una serie de cambios y nuevas propuestas a la educación. Según García Aretio, Ruíz Corbella, Quintanal Díaz, García Blanco y García Pérez (2009, p. 7) "la educación a distancia a pesar de las resistencias sociales, la demanda de este tipo de educación hizo que fuera incorporándose tímidamente, hasta que terminó por imponerse en determinados sectores de la población, especialmente en el campo universitario".

Al respecto, lo expresado por Facundo (2002. p. 10), refuerza lo mencionado por García Aretio (2001), de que las TIC, trae nuevos desafios y oportunidades en esta era de la globalización originando nuevas oportunidades de comunicación; del mismo modo Muñoz (2009), mencionado por (Yarithza y Piñero, 2012, p. 3), en su análisis sobre la socialización expone que ésta se "genera a partir de un proceso de interrelación que emerge en la relación bidireccional entre el individuo y las demás personas que lo rodean, y que ejercen una influencia social modificadora". 
Debemos reconocer que existe una tensión entre tradición y cambio y por la celeridad con que ocurren los cambios sociales, donde imperiosamente las instituciones educativas que se ve forzada a revisar el concepto mismo de educación y el papel que ha venido desempeñando en el proceso de formación social ya que el proceso educativo se da en el escenario de una realidad social concreta y es el factor potenciador de la sociedad, por ser parte integral de ella. En este contexto que la comprensión de la inserción de la teleformación debería partir de la relación fundamental educación-clase social-conceptos y preconceptos que de la misma se tenga. Sobre este punto extraemos lo mencionado por la UNESCO:

Vivimos tiempos de grandes transformaciones tecnológicas que modifican de manera profunda las relaciones humanas. El acceso y generación de conocimiento pasan a ser los motores del desarrollo. Las nuevas formas de conectividad están en el corazón de procesos de cambio en las esferas económicas, políticas y culturales que han dado lugar a lo que se denomina "globalización". Las personas se involucran en nuevas formas de participación, control social y activismo a través de las redes sociales; con ello, las democracias se enriquecen, conformando un nuevo orden mundial en el que surge el ciberciudadano, con más poder del que nunca tuvo el ciudadano convencional. La tecnología digital se hace presente en todas las áreas de actividad y colabora con los cambios que se producen en el trabajo, la familia y la educación, entre otros. (UNESCO, 2014, p.16).

Ante esto, implementar nuevos rumbos a la educación paraguaya, sería una variante y un desafio ante la constante evolución sociocultural en que estamos encaminados y por qué no mencionar que unas de esas variantes sería la de dar impulso en el ámbito de la educación a la modalidad de teleformación, como una búsqueda de innovación de la educación paraguaya y, es quizá una propuesta como alternativa para llegar a zonas geográficas distantes y a grupos laborales que tienen dificultades para ingresar a la universidad convencional.

Empero, tener presente que también esta modalidad formativa plantea una serie de inconvenientes, como son el gran costo inicial de infraestructura, la complejidad del acceso a los recursos, las escasas normas de calidad para la formación, la complejidad en las condiciones de privacidad y seguridad en la red, el esfuerzo que supone usar las herramientas telemáticas para el estudiante y el docente, la falta de hábitos de aprendizaje y enseñanza mediante las herramientas telemáticas, entre otras (Salis y Masili 1997; Marcelo y Lavié, 2000; Marcelo, 2003). 
Como puede observarse, nuestra sociedad va asumida paulatinamente a la tecnología digital como parte de nuestra cultura, ya que ésta ha inducido a innumerables transformaciones y cambios, desde nuestras actividades cotidianas, los sistemas y estructuras educativas, la comunicación personal y comunitaria. Se ha introducido también, dentro de nuestro entorno social y de la cultura misma.

\section{CONCLUSIÓN}

Se puede plantear como hipótesis que los aspectos socioculturales y el nivel de desarrollo de las TIC, tienen una incidencia directa en la implementación de la teleformación en el Paraguay, ante esto y el avance de la era digital a nivel mundial, nuestro país debe evitar estar excluido de este proceso ya que significaría un relegamiento en el camino de ir oxigenando nuestro sistema educativo a nivel superior, con enfoques y modalidades que sirvan de reingenieria a los modelos tradicionales, y también podría agravar la exclusión y/o la brecha sociocultural y tecnológico.

El evolutivo proceso de inserción de la Tecnología de la información y Comunicación en nuestro medio, y sobre todo en la implementación progresiva de la teleformación en el contexto paraguayo, implica no solamente el aspecto económico, político, la disposición de los requerimientos de programas informáticos y/o técnicos sino también conlleva lo sociocultural, referido sobre todo a creencias y realidades.

Las posibilidades que ofrece las TIC, dentro del ámbito educativo es inmensa y dentro de ella incentivar la teleformación y la credibilidad en ella es un desafio y una deuda pendiente para con la sociedad educativa, pues es un medio confiable para acceder a un proceso del conocimiento: aprender habilidades, competencias y actitudes, ampliar relaciones (aspectos estos no diferentes de las presenciales), superar barreras geográficas, sociales y culturales, ahorrar recursos como tiempo y costos de traslado, desarrollar el espíritu de autonomía y autodisciplina, entre otros.

Teniendo como base lo expuesto, podriamos hacer hincapié en la necesidad de realizar un estudio minucioso de las TIC, dentro del contexto espacial, cultural en el que se desenvuelve el ciudadano paraguayo, como así también investigar cómo dentro de esta coyuntura actual, se podría romper las barreras espaciotemporales, buscando acortar esa brecha digital, la cual que redituaría en un desarrollo tecnológico y educativo, necesarios para impulsar en el país. 


\section{REFERENCIAS}

Bogado, Ó. (14/06/2012). Servicio de internet no será eficiente si Paraguay no tiene conexión a red mundial. Ultima Hora Digital: Nacionales. Recuperado de http:/ /www.ultimahora.com/servicio-internet-no-sera-eficiente-si-paraguay-no-tieneconexion-red-mundial-n536723.html

Cernuzzi, L., Vargas, E. A., y González, V. (2008). Educación superior y desarrollo del capital humano. Asunción: CADEP.

Cueva Carrión, S. P., Pacheco Montoya, E. P., Rodríguez Morales, G. R., y Santos Delgado, A. A. (2009). Tecnologias de Información y comunicación (TIC's) en la Educación Superior. Ecuador: Universidad Técnica Particular de Loja.

Dugarte de Villegas, A. y Guanipa, L. (2009). Las TIC: Medios Didácticos en Educación Superior. Valencia, Venezuela: Universidad de Carabobo.

Facundo, A. (2002). La educación superior abierta y a distancia: Necesidades para su establecimiento y desarrollo en América Latina y el Caribe. UNESCO/IESAL. Rev El Tintero, 7, nov.

García Aretio, L., Ruiz Corbella, M., Quintanal Diaz, J., García Blanco, M., y García Pérez, M. (2009). Concepción y tendencias de la educación a distancia en América Latina. Madrid: Centro de Altos Estudios Universitarios de la OEI.

García Aretio, L. (2001). La educación a distancia: De la teoría a la práctica. Barcelona: Ariel.

GATE (Gabinete de Tele-educación). (1998). Informe sobre tele-educación en la formación de Postgrado. Madrid: Universidad Politécnica de Madrid (UPM).

Goñi, J. J. (2005). Evolución de la TIC: Oportunidades y amenazas sociales. Recuperado de http://info.ibermaticacloud.com/ebooks/Evolucion/21/files/assets/seo/page14.html

Harris, M. (1990). Antropología cultural. Madrid: Alianza Editorial.

Hughes, G. (2007). Liderazgo. India: McGraw-Hill.

Iriarte Denis, I. (2015, 13 enero). Internet móvil de Paraguay, entre los más lentos de Sudamérica. Periódico digital 5 Días. Recuperado de http://www.5dias.com.py/37913internet-movil-de-paraguay-entre-los-mas-lentos-de-sudamerica

Ley $N^{\circ} 4995$ de Educación Superior. Poder Legislativo. Asunción, Paraguay, 02 de Agosto del 2013.

Maldonado López, L. M. (2007). Las tecnologías de información y comunicación un recurso estratégico para la educación superior. Visión Gerencial, 6 (2): 257-268. Recuperado de http://erevistas.saber.ula.ve/index.php/visiongerencial/article/view/1014

Marcelo, C. y Lavié, J. M. (2000). Formación y nuevas tecnologías: Posibilidades y condiciones de la teleformación como espacio de aprendizaje. Bordón, 52, 3, 385-406.

Marcelo, C. (2003). Experto en e-learning: Curso de experto. Sevilla: Universidad de Sevilla.

Marqués Graells, P. (2000). Impacto de las TIC en educación: Funciones y limitaciones. Recuperado de http://www.peremarques.net/si.htm

Martin, J. M. (2012). Integración de las TIC en la educación escolar paraguaya. Asunción: Universidad Autónoma de Asunción.

Martinez Sánchez, F. (2001). El profesorado ante las nuevas Tecnologias. Murcia: Universidad de Murcia.

Martinez Serrano, E., y Serrano Santoyo, A. (2007). La evolución hacia una nueva brecha digital. Recuperado de http://www.labrechadigital.org/labrecha 
Muñoz, J. (2009). La importancia de la socialización en la educación actual. Recuperado de http://www.csicsif.es/andalucia/modules/mod_ense/revista/pdf/Numero_14/JOSE\%20 MARIA_MUNOZ_1.pdf

ONU (Organización de las Naciones Unidas). (2014). Tecnologías de la información y las comunicaciones para un desarrollo social y económico incluyente. Ginebra: Comisión de Ciencia y Tecnología para el Desarrollo.

Navales, M., Omaña, O., y Perazzo, C. (2000). Las tecnologías de la información y la comunicación y su impacto en la educación. Hidalgo: Universidad Autónoma del Estado de Hidalgo. Recuperado de http://bibliotecadigital.conevyt.org.mx/colecciones/docu mentos/somece/43.pdf

Paraguay, con internet lentísimo. (20/02/2014). ABC color, nacionales. Recuperado de http://www.abc.com.py/nacionales/internet-en-paraguay-entre-los-mas-lentos-1217005.html

Peterssen Nodarse, G. (2001). Perspectivas de la enseñanza virtual por internet en América Latina. Recuperado de http://www.espectador.com/text/documentos/doc05177.html

Robles, E. (2003). Cultura y era tecnológica. Revista Digital electrónica Razón y Palabra, 35. Recuperado de http://razonypalabra.org.mx/anteriores/n35/erobles.html\#Er

Rojas, M. (2013). Propuesta de politicas públicas en Tecnologías de la Información y Comunicación (TICs). Paraguay en debate. Recuperado de http://www.cird.org.py/comunicacion/documentos/04TICS.pdf

Sáez Vacas, F. (1997). Innovación tecnológica y reingeniería en los procesos educativos. En Alonso, C. (coord.). La tecnología educativa a finales del siglo XX: Concepciones, conexiones y limites con otras asignaturas. Barcelona: Eumo-Grafic.

Salis, C., y Masili, G. (1997). The user control on verbal/non-verbal knowledge visualization. Technology and Teacher Education Annual. Recuperado de http:/ / reposital.cuaed.unam.mx:8080/jspui/bitstream/123456789/2554/1/128.pdf

UNESCO. (1998). Conferencia mundial sobre la educación superior en el Siglo XXI: Visión y acción. Recuperado de http://unesdoc.unesco.org/images/0011/001136/113602 So.pdf

UNESCO. (2014). Enfoques estratégicos sobre las TIC en educación en América Latina y el Caribe. Santiago de Chile: OREALC/UNESCO.

Urkola Carrera, L. (2006). Análisis del perfil formativo en tecnologías de la información y comunicación del alumnado universitario y su adecuación a las necesidades empresariales en el ámbito de la CAPV. Barcelona: Euskal Herriko Unibertsitateko Argitalpen Zerbitzua.

Wild, M. (1999). Culture and new technologies. British Journal of Educational Technology, 30. (3), 195-199.

Yarithza, A., y Piñero L. (2012). Dimensionalidades de las TIC en la socialización del conocimiento en contextos educativos, área temática: Tendencias investigativas en TIC. (Memorias del Congreso Internacional TIC y Pedagogía. III edición. Universidad Pedagógica Experimental Libertador. Instituto Pedagógico de Barquisimeto "Luis Beltrán Prieto Figueroa", Colombia, 16, 17 y 18 de mayo de 2012). 